\title{
Expressions for values of the gamma function
}

\author{
Raimundas Vidūnas* \\ Kyushu University
}

February 1, 2008

\begin{abstract}
This paper presents expressions for gamma values at rational points with the denominator dividing 24 or 60 . These gamma values are expressed in terms of 10 distinct gamma values and rational powers of $\pi$ and a few real algebraic numbers. Our elementary list of formulas can be conveniently used to evaluate, for example, algebraic Gauss hypergeometric functions by the Gauss identity. Also, algebraic independence of gamma values and their relation to the elliptic $\mathbf{K}$ function are briefly discussed.
\end{abstract}

\section{Introduction}

The gamma function AAR, Chapter 1] satisfies the difference equation

$$
\Gamma(x+1)=x \Gamma(x)
$$

the Euler reflection formula

$$
\Gamma(x) \Gamma(1-x)=\frac{\pi}{\sin (\pi x)},
$$

and the Gauss multiplication formula

$$
\Gamma(x) \Gamma\left(x+\frac{1}{n}\right) \ldots \Gamma\left(x+\frac{n-1}{n}\right)=n^{\frac{1}{2}-n x}(2 \pi)^{\frac{n-1}{2}} \Gamma(n x) .
$$

In the last formula, $n$ is a positive integer. Its special case $n=2$ is known as Legendre's duplication formula. We refer to these functional equations for the gamma function as the standard equations.

Values of the gamma function at rational points are of broad interest. By historical motivation, $\Gamma(n)=(n-1)$ ! when $n$ is a positive integer. An easy consequence of the reflection formula is $\Gamma\left(\frac{1}{2}\right)=\sqrt{\pi}$. By using difference equation (10) one can evaluate $\Gamma(x)$

*Supported by the 21 Century COE Programme "Development of Dynamic Mathematics with High Functionality" of the Ministry of Education, Culture, Sports, Science and Technology of Japan. 
for rational $x$ with the denominator 2. No explicit evaluations of other gamma values are known. Some gamma terms (i.e., quotients of products of gamma values) occur as values of hypergeometric functions at special points [AAR] and as period integrals [KZ, De. In particular, this applies to values of the elliptic $\mathbf{K}$-function at so-called elliptic integral singular values $[\mathbf{Z u}],[\mathrm{SC}],[\mathrm{Ca}]$. Conversely, some gamma values at rational points can be expressed in terms of elliptic integrals; see [BZ, Wa1 and Section [5 in this paper.

The purpose of this paper is to present explicit relations between gamma values in the set

$$
\left\{\Gamma\left(\frac{k}{n}\right) \mid k, n \in \mathbf{Z} ; 0<\frac{k}{n}<1 ; n \text { divides } 24 \text { or } 60\right\} .
$$

We show that standard formulas (2)-(3) imply that all these gamma values can be multiplicatively expressed in terms of rational powers of $\pi$, rational powers of few algebraic numbers, and the following 10 gamma values:

$$
\begin{aligned}
& \Gamma\left(\frac{1}{3}\right), \quad \Gamma\left(\frac{1}{4}\right), \quad \Gamma\left(\frac{1}{5}\right), \quad \Gamma\left(\frac{2}{5}\right), \quad \Gamma\left(\frac{1}{8}\right), \\
& \Gamma\left(\frac{1}{15}\right), \quad \Gamma\left(\frac{1}{20}\right), \quad \Gamma\left(\frac{1}{24}\right), \quad \Gamma\left(\frac{1}{60}\right), \quad \Gamma\left(\frac{7}{60}\right) \text {. }
\end{aligned}
$$

In Section 2 we present explicit expressions for the gamma values in (4) in these terms. By using difference equation (11) and our list of formulas, one can express any gamma value at a rational point with the denominator dividing 24 or 60 in the same fashion. In Section 3 we indicate an elementary proof of our evaluations, and show that the standard formulas do not imply any relations between the distinguished values in (5). If Lang's conjecture [La] is true, those 10 gamma values and the constant $\pi$ are algebraically independent over $\mathbf{Q}$.

Relations between gamma values at rational points with small denominators are widely known; see [WR page GammaFunction.html] for example. Some tricky relations between gamma values from the set (4) are derived in [BZ, [Ka and probably by other authors. Of special interest are gamma terms with algebraic values $[\mathrm{KO}$, [Da]. Our list of relations between gamma values may be useful for many purposes. For example, explicit evaluation of algebraic Gauss hypergeometric functions at $x=1$ requires gamma values at rational points with the denominators dividing 24 or 60 ; see Section 4 Our formula list is precisely enough for this purpose. In Section 5 we present available expressions of gamma values in (5) in terms of the elliptic $\mathbf{K}$-function.

\section{Explicit formulas}

Here we present a list of explicit expressions of gamma values in the set (4) in terms of the gamma values in (5). A proof of these relations is indicated in the following Section. A comparable list of expressions for gamma values is available at [Koo]. 
To make formulas more compact, we introduce the following constants:

$$
\begin{array}{lll}
\phi=5+\sqrt{5}, & \phi^{\star}=5-\sqrt{5}, \\
\psi=\sqrt{5+2 \sqrt{5},} & \psi^{\star}=\sqrt{5-2 \sqrt{5} .}
\end{array}
$$

Note that

$$
\phi \phi^{\star}=20, \quad \psi \psi^{\star}=\sqrt{5}, \quad \psi=\frac{\phi^{3 / 2}}{2^{3 / 2} \sqrt{5}}, \quad \psi^{\star}=\frac{\phi^{\star} 3 / 2}{2^{3 / 2} \sqrt{5}} .
$$

We express gamma values from (4) in terms of the values in (5) and rational powers of the following constants:

$$
\begin{aligned}
& \pi, \quad 2, \quad 3, \quad 5, \quad \sqrt{2} \pm 1, \quad \sqrt{3} \pm 1, \quad \sqrt{3} \pm \sqrt{2}, \quad \sqrt{5} \pm \sqrt{3}, \\
& \phi, \quad \phi^{\star}, \quad \sqrt{15} \pm \psi, \quad \sqrt{15} \pm \psi^{\star}, \quad \sqrt{10} \pm \sqrt{\phi}, \quad \sqrt{10} \pm \sqrt{\phi^{\star}} .
\end{aligned}
$$

When applying our formulas to gamma terms, it is easy to invert and simplify these algebraic numbers. Some useful expressions with these algebraic numbers are presented in Lemma 3.2 in the next Section.

Here is our list of formulas:

$$
\begin{array}{rlrl}
\Gamma\left(\frac{1}{2}\right) & =\sqrt{\pi}, & \Gamma\left(\frac{2}{3}\right) & =\frac{2 \pi}{\sqrt{3}} \Gamma\left(\frac{1}{3}\right)^{-1}, \\
\Gamma\left(\frac{3}{4}\right) & =\pi \sqrt{2} \Gamma\left(\frac{1}{4}\right)^{-1}, & \Gamma\left(\frac{1}{6}\right) & =\frac{\sqrt{3}}{\sqrt{\pi} 2^{1 / 3}} \Gamma\left(\frac{1}{3}\right)^{2}, \\
\Gamma\left(\frac{3}{5}\right) & =\frac{\pi \sqrt{2} \sqrt{\phi^{\star}}}{\sqrt{5}} \Gamma\left(\frac{2}{5}\right)^{-1}, & \Gamma\left(\frac{5}{6}\right) & =\frac{\pi^{3 / 2} 2^{4 / 3}}{\sqrt{3}} \Gamma\left(\frac{1}{3}\right)^{-2}, \\
\Gamma\left(\frac{4}{5}\right) & =\frac{\pi \sqrt{2} \sqrt{\phi}}{\sqrt{5}} \Gamma\left(\frac{1}{5}\right)^{-1}, & \Gamma\left(\frac{3}{8}\right) & =\sqrt{\pi} \sqrt{\sqrt{2}-1} \Gamma\left(\frac{1}{4}\right)^{-1} \Gamma\left(\frac{1}{8}\right), \\
\Gamma\left(\frac{5}{8}\right) & =\sqrt{\pi} 2^{3 / 4} \Gamma\left(\frac{1}{4}\right) \Gamma\left(\frac{1}{8}\right)^{-1}, & \Gamma\left(\frac{7}{8}\right) & =\pi 2^{3 / 4} \sqrt{\sqrt{2}+1} \Gamma\left(\frac{1}{8}\right)^{-1}, \\
\Gamma\left(\frac{1}{10}\right) & =\frac{\sqrt{\phi}}{\sqrt{\pi} 2^{7 / 10}} \Gamma\left(\frac{1}{5}\right)^{-1} \Gamma\left(\frac{2}{5}\right), & \Gamma\left(\frac{3}{10}\right) & =\frac{\sqrt{\pi} \phi^{\star}}{2^{3 / 5} \sqrt{5}} \Gamma\left(\frac{1}{5}\right) \Gamma\left(\frac{2}{5}\right)^{-1}, \\
\Gamma\left(\frac{7}{10}\right) & =\sqrt{\pi} 2^{3 / 5} \Gamma\left(\frac{1}{5}\right)^{-1} \Gamma\left(\frac{2}{5}\right), & \Gamma\left(\frac{9}{10}\right) & =\frac{\pi^{3 / 2} 2^{7 / 10} \sqrt{\phi}}{\sqrt{5}} \Gamma\left(\frac{1}{5}\right)^{-1} \Gamma\left(\frac{2}{5}\right)^{-1}, \\
\Gamma\left(\frac{1}{12}\right) & =\frac{3^{3 / 8} \sqrt{\sqrt{3}+1}}{\sqrt{\pi} 2^{1 / 4}} \Gamma\left(\frac{1}{3}\right) \Gamma\left(\frac{1}{4}\right), & & \\
\Gamma\left(\frac{5}{12}\right) & =\frac{\sqrt{\pi} 2^{1 / 4} \sqrt{\sqrt{3}-1} \Gamma\left(\frac{1}{4}\right) \Gamma\left(\frac{1}{3}\right)^{-1},}{3^{1 / 8}}, \\
\Gamma\left(\frac{7}{12}\right) & =\sqrt{\pi} 2^{1 / 4} 3^{1 / 8} \sqrt{\sqrt{3}-1} \Gamma\left(\frac{1}{3}\right) \Gamma\left(\frac{1}{4}\right)^{-1},
\end{array}
$$




$$
\begin{aligned}
& \Gamma\left(\frac{11}{12}\right)=\frac{\pi^{3 / 2} 2^{3 / 4} \sqrt{\sqrt{3}+1}}{3^{3 / 8}} \Gamma\left(\frac{1}{3}\right)^{-1} \Gamma\left(\frac{1}{4}\right)^{-1}, \\
& \Gamma\left(\frac{2}{15}\right)=\frac{\sqrt{\phi^{\star}} \sqrt{\sqrt{15}-\psi^{\star}}}{2 \cdot 3^{7 / 20} 5^{1 / 3}} \Gamma\left(\frac{1}{3}\right)^{-1} \Gamma\left(\frac{2}{5}\right) \Gamma\left(\frac{1}{15}\right), \\
& \Gamma\left(\frac{4}{15}\right)=\frac{\sqrt{\phi} \sqrt{\sqrt{15}-\psi} \sqrt{\sqrt{15}-\psi^{\star}}}{2^{3 / 2} 3^{3 / 10} \sqrt{5}} \Gamma\left(\frac{1}{5}\right)^{-1} \Gamma\left(\frac{2}{5}\right) \Gamma\left(\frac{1}{15}\right), \\
& \Gamma\left(\frac{7}{15}\right)=\frac{3^{9 / 20} \sqrt{\phi^{\star}} \sqrt{\sqrt{15}+\psi^{\star}}}{2 \cdot 5^{1 / 6}} \Gamma\left(\frac{1}{3}\right) \Gamma\left(\frac{1}{5}\right) \Gamma\left(\frac{1}{15}\right)^{-1}, \\
& \Gamma\left(\frac{8}{15}\right)=\frac{\pi \sqrt{2} \sqrt{\sqrt{15}-\psi}}{3^{9 / 20} 5^{1 / 3}} \Gamma\left(\frac{1}{3}\right)^{-1} \Gamma\left(\frac{1}{5}\right)^{-1} \Gamma\left(\frac{1}{15}\right), \\
& \Gamma\left(\frac{11}{15}\right)=2 \pi \cdot 3^{3 / 10} \Gamma\left(\frac{1}{5}\right) \Gamma\left(\frac{2}{5}\right)^{-1} \Gamma\left(\frac{1}{15}\right)^{-1}, \\
& \Gamma\left(\frac{13}{15}\right)=\frac{\pi \sqrt{2} 3^{7 / 20} \sqrt{\sqrt{15}+\psi}}{5^{1 / 6}} \Gamma\left(\frac{1}{3}\right) \Gamma\left(\frac{2}{5}\right)^{-1} \Gamma\left(\frac{1}{15}\right)^{-1}, \\
& \Gamma\left(\frac{14}{15}\right)=\frac{\pi \sqrt{\phi} \sqrt{\sqrt{15}+\psi} \sqrt{\sqrt{15}+\psi^{\star}}}{\sqrt{2} \sqrt{5}} \Gamma\left(\frac{1}{15}\right)^{-1}, \\
& \Gamma\left(\frac{3}{20}\right)=\frac{\sqrt{\pi} \phi^{\star} \sqrt{\sqrt{10}-\sqrt{\phi^{\star}}}}{2^{21 / 20} 5^{7 / 8}} \Gamma\left(\frac{2}{5}\right)^{-1} \Gamma\left(\frac{1}{20}\right), \\
& \Gamma\left(\frac{7}{20}\right)=\frac{\sqrt{\pi} \sqrt{\sqrt{10}-\sqrt{\phi}}}{2^{3 / 20} 5^{3 / 8}} \Gamma\left(\frac{1}{5}\right)^{-1} \Gamma\left(\frac{1}{20}\right), \\
& \Gamma\left(\frac{9}{20}\right)=\frac{\pi \sqrt{\sqrt{10}-\sqrt{\phi}} \sqrt{\sqrt{10}-\sqrt{\phi^{\star}}}}{2^{1 / 5} \sqrt{5}} \Gamma\left(\frac{1}{5}\right)^{-1} \Gamma\left(\frac{2}{5}\right)^{-1} \Gamma\left(\frac{1}{20}\right), \\
& \Gamma\left(\frac{11}{20}\right)=2^{1 / 5} \sqrt{\phi} \Gamma\left(\frac{1}{5}\right) \Gamma\left(\frac{2}{5}\right) \Gamma\left(\frac{1}{20}\right)^{-1}, \\
& \Gamma\left(\frac{13}{20}\right)=\frac{\sqrt{\pi} 2^{3 / 20} \sqrt{\phi^{\star}} \sqrt{\sqrt{10}+\sqrt{\phi^{\star}}}}{5^{1 / 8}} \Gamma\left(\frac{1}{5}\right) \Gamma\left(\frac{1}{20}\right)^{-1}, \\
& \Gamma\left(\frac{17}{20}\right)=\frac{\sqrt{\pi} 2^{1 / 20} \sqrt{\phi} \sqrt{\sqrt{10}+\sqrt{\phi}}}{5^{1 / 8}} \Gamma\left(\frac{2}{5}\right) \Gamma\left(\frac{1}{20}\right)^{-1}, \\
& \Gamma\left(\frac{19}{20}\right)=\frac{\pi \sqrt{\phi} \sqrt{\sqrt{10}+\sqrt{\phi}} \sqrt{\sqrt{10}+\sqrt{\phi^{\star}}}}{\sqrt{5}} \Gamma\left(\frac{1}{20}\right)^{-1}, \\
& \Gamma\left(\frac{5}{24}\right)=\frac{\sqrt{\pi} \sqrt{\sqrt{2}-1} \sqrt{\sqrt{3}-1}}{2^{1 / 6} \sqrt{3}} \Gamma\left(\frac{1}{3}\right)^{-1} \Gamma\left(\frac{1}{24}\right), \\
& \Gamma\left(\frac{7}{24}\right)=\frac{\sqrt{\pi} \sqrt{\sqrt{3}-1} \sqrt{\sqrt{3}-\sqrt{2}}}{2^{1 / 4} 3^{3 / 8}} \Gamma\left(\frac{1}{4}\right)^{-1} \Gamma\left(\frac{1}{24}\right),
\end{aligned}
$$




$$
\begin{aligned}
& \Gamma\left(\frac{11}{24}\right)=\frac{\pi 2^{1 / 12} \sqrt{\sqrt{2}-1} \sqrt{\sqrt{3}-\sqrt{2}}}{3^{3 / 8}} \Gamma\left(\frac{1}{3}\right)^{-1} \Gamma\left(\frac{1}{4}\right)^{-1} \Gamma\left(\frac{1}{24}\right), \\
& \Gamma\left(\frac{13}{24}\right)=2^{2 / 3} 3^{3 / 8} \sqrt{\sqrt{3}+1} \Gamma\left(\frac{1}{3}\right) \Gamma\left(\frac{1}{4}\right) \Gamma\left(\frac{1}{24}\right)^{-1}, \\
& \Gamma\left(\frac{17}{24}\right)=2 \sqrt{\pi} 3^{3 / 8} \sqrt{\sqrt{2}+1} \Gamma\left(\frac{1}{4}\right) \Gamma\left(\frac{1}{24}\right)^{-1}, \\
& \Gamma\left(\frac{19}{24}\right)=\sqrt{\pi} 2^{11 / 12} \sqrt{3} \sqrt{\sqrt{3}+\sqrt{2}} \Gamma\left(\frac{1}{3}\right) \Gamma\left(\frac{1}{24}\right)^{-1}, \\
& \Gamma\left(\frac{23}{24}\right)=\pi 2^{3 / 4} \sqrt{\sqrt{2}+1} \sqrt{\sqrt{3}+1} \sqrt{\sqrt{3}+\sqrt{2}} \Gamma\left(\frac{1}{24}\right)^{-1}, \\
& \Gamma\left(\frac{1}{30}\right)=\frac{3^{9 / 20} \sqrt{\phi} \sqrt{\sqrt{15}+\psi}}{\sqrt{\pi} 2^{16 / 15} 5^{1 / 6}} \Gamma\left(\frac{1}{3}\right) \Gamma\left(\frac{1}{5}\right), \\
& \Gamma\left(\frac{7}{30}\right)=\frac{3^{3 / 20} \sqrt{\phi^{\star}} \sqrt{\sqrt{15}+\psi^{\star}}}{\sqrt{\pi} 2^{22 / 15} 5^{1 / 6}} \Gamma\left(\frac{1}{3}\right) \Gamma\left(\frac{2}{5}\right), \\
& \Gamma\left(\frac{11}{30}\right)=\frac{\sqrt{\pi} \sqrt{\phi} \sqrt{\sqrt{15}-\psi}}{2^{11 / 15} 3^{1 / 20} 5^{1 / 3}} \Gamma\left(\frac{1}{3}\right)^{-1} \Gamma\left(\frac{1}{5}\right), \\
& \Gamma\left(\frac{13}{30}\right)=\frac{\sqrt{\pi} 3^{7 / 20} \phi^{\star} \sqrt{\sqrt{15}-\psi^{\star}}}{2^{41 / 30} 5^{2 / 3}} \Gamma\left(\frac{1}{3}\right) \Gamma\left(\frac{2}{5}\right)^{-1}, \\
& \Gamma\left(\frac{17}{30}\right)=\frac{\sqrt{\pi} \sqrt{\phi^{\star}} \sqrt{\sqrt{15}-\psi^{\star}}}{2^{2 / 15} 3^{7 / 20} 5^{1 / 3}} \Gamma\left(\frac{1}{3}\right)^{-1} \Gamma\left(\frac{2}{5}\right), \\
& \Gamma\left(\frac{19}{30}\right)=\frac{\sqrt{\pi} 3^{1 / 20} \phi \sqrt{\sqrt{15}-\psi}}{2^{23 / 30} 5^{2 / 3}} \Gamma\left(\frac{1}{3}\right) \Gamma\left(\frac{1}{5}\right)^{-1}, \\
& \Gamma\left(\frac{23}{30}\right)=\frac{\pi^{3 / 2} \phi^{\star} \sqrt{\sqrt{15}+\psi^{\star}}}{2^{1 / 30} 3^{3 / 20} 5^{5 / 6}} \Gamma\left(\frac{1}{3}\right)^{-1} \Gamma\left(\frac{2}{5}\right)^{-1}, \\
& \Gamma\left(\frac{29}{30}\right)=\frac{\pi^{3 / 2} \phi \sqrt{\sqrt{15}+\psi}}{2^{13 / 30} 3^{9 / 20} 5^{5 / 6}} \Gamma\left(\frac{1}{3}\right)^{-1} \Gamma\left(\frac{1}{5}\right)^{-1}, \\
& \Gamma\left(\frac{11}{60}\right)=\frac{\sqrt{\pi} \sqrt{\phi} \sqrt{\sqrt{15}-\psi} \sqrt{\sqrt{10}-\sqrt{\phi}}}{2^{5 / 4} \sqrt{3} 5^{17 / 24}} \Gamma\left(\frac{1}{3}\right)^{-1} \Gamma\left(\frac{1}{60}\right), \\
& \Gamma\left(\frac{13}{60}\right)=\frac{\sqrt{\pi} \sqrt{\phi^{\star}} \sqrt{\sqrt{3}+1} \sqrt{\sqrt{5}-\sqrt{3}} \sqrt{\sqrt{15}-\psi^{\star}}}{2^{13 / 10} 3^{3 / 20} 5^{3 / 8}} \Gamma\left(\frac{2}{5}\right)^{-1} \Gamma\left(\frac{7}{60}\right), \\
& \Gamma\left(\frac{17}{60}\right)=\frac{\sqrt{\pi} \sqrt{\phi^{\star}} \sqrt{\sqrt{15}-\psi^{\star}} \sqrt{\sqrt{10}-\sqrt{\phi^{\star}}}}{2^{3 / 4} \sqrt{3} 5^{11 / 24}} \Gamma\left(\frac{1}{3}\right)^{-1} \Gamma\left(\frac{7}{60}\right), \\
& \Gamma\left(\frac{19}{60}\right)=\frac{\sqrt{\pi} \sqrt{\phi} \sqrt{\sqrt{3}-1} \sqrt{\sqrt{5}-\sqrt{3}} \sqrt{\sqrt{15}-\psi}}{2^{7 / 5} 3^{9 / 20} 5^{5 / 8}} \Gamma\left(\frac{1}{5}\right)^{-1} \Gamma\left(\frac{1}{60}\right),
\end{aligned}
$$




$$
\begin{aligned}
& \Gamma\left(\frac{23}{60}\right)=\frac{\pi \sqrt{\phi^{\star}} \sqrt{\sqrt{3}+1} \sqrt{\sqrt{5}-\sqrt{3}} \sqrt{\sqrt{10}-\sqrt{\phi^{\star}}}}{2^{11 / 20} 3^{3 / 20} 5^{7 / 12}} \Gamma\left(\frac{1}{3}\right)^{-1} \Gamma\left(\frac{2}{5}\right)^{-1} \Gamma\left(\frac{7}{60}\right), \\
& \Gamma\left(\frac{29}{60}\right)=\frac{\pi \sqrt{\phi} \sqrt{\sqrt{3}-1} \sqrt{\sqrt{5}-\sqrt{3}} \sqrt{\sqrt{10}-\sqrt{\phi}}}{2^{23 / 20} 3^{9 / 20} 5^{7 / 12}} \Gamma\left(\frac{1}{3}\right)^{-1} \Gamma\left(\frac{1}{5}\right)^{-1} \Gamma\left(\frac{1}{60}\right) \text {, } \\
& \Gamma\left(\frac{31}{60}\right)=\frac{3^{9 / 20} \sqrt{\phi} \sqrt{\sqrt{15}+\psi}}{2^{1 / 10} 5^{1 / 6}} \Gamma\left(\frac{1}{3}\right) \Gamma\left(\frac{1}{5}\right) \Gamma\left(\frac{1}{60}\right)^{-1}, \\
& \Gamma\left(\frac{37}{60}\right)=\frac{3^{3 / 20} \sqrt{\phi^{\star}} \sqrt{\sqrt{15}+\psi^{\star}}}{2^{7 / 10} 5^{1 / 6}} \Gamma\left(\frac{1}{3}\right) \Gamma\left(\frac{2}{5}\right) \Gamma\left(\frac{7}{60}\right)^{-1}, \\
& \Gamma\left(\frac{41}{60}\right)=\frac{\sqrt{\pi} 2^{3 / 20} 3^{9 / 20} \sqrt{\phi} \sqrt{\sqrt{10}+\sqrt{\phi}}}{5^{1 / 8}} \Gamma\left(\frac{1}{5}\right)^{-1} \Gamma\left(\frac{1}{60}\right), \\
& \Gamma\left(\frac{43}{60}\right)=\frac{\sqrt{\pi} \sqrt{3} \sqrt{\phi^{\star}} \sqrt{\sqrt{3}-1} \sqrt{\sqrt{5}+\sqrt{3}}}{\sqrt{2} 5^{7 / 24}} \Gamma\left(\frac{1}{3}\right) \Gamma\left(\frac{7}{60}\right)^{-1}, \\
& \Gamma\left(\frac{47}{60}\right)=\frac{\sqrt{\pi} 2^{1 / 20} 3^{3 / 20} \sqrt{\phi^{\star}} \sqrt{\sqrt{10}+\sqrt{\phi^{\star}}}}{5^{3 / 8}} \Gamma\left(\frac{2}{5}\right)^{-1} \Gamma\left(\frac{7}{60}\right), \\
& \Gamma\left(\frac{49}{60}\right)=\frac{\sqrt{\pi} \sqrt{3} \sqrt{\phi} \sqrt{\sqrt{3}+1} \sqrt{\sqrt{5}+\sqrt{3}}}{5^{1 / 24}} \Gamma\left(\frac{1}{3}\right) \Gamma\left(\frac{1}{60}\right)^{-1}, \\
& \Gamma\left(\frac{53}{60}\right)=\frac{\pi \phi^{\star} \sqrt{\sqrt{3}-1} \sqrt{\sqrt{5}+\sqrt{3}} \sqrt{\sqrt{15}+\psi^{\star}} \sqrt{\sqrt{10}+\sqrt{\phi^{\star}}}}{2^{5 / 4} 5^{3 / 4}} \Gamma\left(\frac{7}{60}\right)^{-1}, \\
& \Gamma\left(\frac{59}{60}\right)=\frac{\pi \phi \sqrt{\sqrt{3}+1} \sqrt{\sqrt{5}+\sqrt{3}} \sqrt{\sqrt{15}+\psi} \sqrt{\sqrt{10}+\sqrt{\phi}}}{2^{5 / 4} 5^{3 / 4}} \Gamma\left(\frac{1}{60}\right)^{-1} .
\end{aligned}
$$

\section{Proof of the formulas}

Here we give an elementary proof of all identities in the previous Section. We do not reproduce detailed computations in our proofs; they are quite straightforward though tedious. Lemma 3.2 gives compact expressions for some products of employed algebraic numbers (8). We also show that the standard equations do not imply any relations between gamma values in (5), and discuss briefly algebraic independence of those gamma values. Along the way, we give a set of 16 gamma values which are enough to express any gamma value at a rational point with the denominator dividing 120 .

First we indicate relevant values of the sine function. To read off the sinus values, one has to compare formulas in Lemma 3.1] with the following form of Euler's identity:

$$
\exp (i x)=\sin \left(\frac{\pi}{2}-x\right)+i \sin x
$$

For completeness, recall the well-known values $\exp \left(\frac{i \pi}{3}\right)=\frac{1+i \sqrt{3}}{2}$ and $\exp \left(\frac{i \pi}{4}\right)=\frac{1+i}{\sqrt{2}}$. 
Lemma 3.1 With the same notation as in ([6), the following formulas hold:

$$
\begin{aligned}
& \exp \left(\frac{i \pi}{5}\right)=\phi \frac{1+i \psi^{\star}}{4 \sqrt{5}}, \quad \exp \left(\frac{2 i \pi}{5}\right)=\phi^{\star} \frac{1+i \psi}{4 \sqrt{5}} \\
& \exp \left(\frac{i \pi}{8}\right)=\frac{\sqrt{\sqrt{2}+1}+i \sqrt{\sqrt{2}-1}}{2^{3 / 4}}, \quad \exp \left(\frac{i \pi}{12}\right)=\frac{\sqrt{3}+1+i(\sqrt{3}-1)}{2 \sqrt{2}}, \\
& \exp \left(\frac{i \pi}{15}\right)=\phi^{\star} \frac{\sqrt{3} \psi+1+i(\psi-\sqrt{3})}{8 \sqrt{5}}, \quad \exp \left(\frac{2 i \pi}{15}\right)=\phi \frac{\sqrt{3} \psi^{\star}+1+i\left(\psi^{\star}-\sqrt{3}\right)}{8 \sqrt{5}}, \\
& \exp \left(\frac{4 i \pi}{15}\right)=\phi^{\star} \frac{\sqrt{3} \psi-1+i(\psi+\sqrt{3})}{8 \sqrt{5}}, \quad \exp \left(\frac{7 i \pi}{15}\right)=\phi \frac{\sqrt{3} \psi^{\star}-1+i\left(\sqrt{3}-\psi^{\star}\right)}{8 \sqrt{5}} \\
& \exp \left(\frac{i \pi}{20}\right)=\sqrt{\phi^{\star}} \frac{\psi+\sqrt{5}+i(\psi-\sqrt{5})}{4 \sqrt{5}}, \quad \exp \left(\frac{3 i \pi}{20}\right)=\sqrt{\phi} \frac{\sqrt{5}+\psi^{\star}+i\left(\sqrt{5}-\psi^{\star}\right)}{4 \sqrt{5}}, \\
& \exp \left(\frac{i \pi}{24}\right)=\frac{\sqrt{2 \sqrt{2}+\sqrt{3}+1}+i \sqrt{2 \sqrt{2}-\sqrt{3}-1}}{2^{5 / 4}} \\
& \exp \left(\frac{7 i \pi}{24}\right)=\frac{\sqrt{2 \sqrt{2}-\sqrt{3}+1}+i \sqrt{2 \sqrt{2}+\sqrt{3}-1}}{2^{5 / 4}}, \\
& \exp \left(\frac{i \pi}{60}\right)=\phi^{\star} \frac{(\sqrt{3}+1)(\psi-\sqrt{3}+2)+i(\sqrt{3}-1)(\sqrt{3}-\psi+2)}{8 \sqrt{10}}, \\
& \exp \left(\frac{7 i \pi}{60}\right)=\phi \frac{(\sqrt{3}-1)\left(\psi^{\star}+\sqrt{3}+2\right)+i(\sqrt{3}+1)\left(\sqrt{3}+\psi^{\star}-2\right)}{8 \sqrt{10}}, \\
& \exp \left(\frac{13 i \pi}{60}\right)=\phi \frac{(\sqrt{3}+1)\left(\psi^{\star}-\sqrt{3}+2\right)+i(\sqrt{3}-1)\left(\sqrt{3}-\psi^{\star}+2\right)}{8 \sqrt{10}}, \\
& \exp \left(\frac{19 i \pi}{60}\right)=\phi^{\star} \frac{(\sqrt{3}-1)(\psi+\sqrt{3}+2)+i(\sqrt{3}+1)(\sqrt{3}+\psi-2)}{8 \sqrt{10}} .
\end{aligned}
$$

Proof. One can deduce the first two formulas by checking the sign of real and imaginary parts on their right-hand sides, and checking the respective equations $x^{5} \pm 1=0$. The remaining formulas can be consequently deduced by inspecting the values of

$$
\begin{array}{r}
\exp \left(\frac{i \pi}{8}\right)^{2}, \exp \left(\frac{i \pi}{12}\right) \exp \left(\frac{i \pi}{4}\right), \exp \left(\frac{i \pi}{15}\right) \exp \left(\frac{i \pi}{3}\right), \exp \left(\frac{i \pi}{15}\right)^{2}, \exp \left(\frac{2 i \pi}{15}\right)^{2}, \\
\exp \left(\frac{2 i \pi}{15}\right) \exp \left(\frac{i \pi}{3}\right), \quad \exp \left(\frac{i \pi}{20}\right) \exp \left(\frac{i \pi}{5}\right), \quad \exp \left(\frac{3 i \pi}{20}\right) \exp \left(\frac{i \pi}{4}\right), \\
\exp \left(\frac{i \pi}{24}\right)^{2}, \quad \exp \left(\frac{i \pi}{24}\right) \exp \left(\frac{i \pi}{4}\right), \quad \exp \left(\frac{i \pi}{60}\right) \exp \left(\frac{i \pi}{4}\right), \\
\exp \left(\frac{i \pi}{12}\right) \exp \left(\frac{i \pi}{5}\right), \quad \exp \left(\frac{i \pi}{60}\right) \exp \left(\frac{i \pi}{5}\right), \quad \exp \left(\frac{7 i \pi}{60}\right) \exp \left(\frac{i \pi}{5}\right) .
\end{array}
$$

Simplification of each exponential expression relates the respective exponent value to earlier concluded exponent values, so it is enough to check those relations with substituted algebraic 
expressions (and sometimes the sign of the new exponent value).

Now we present some expressions with employed algebraic numbers. They can be used to simplify evaluated gamma terms and to transform the sinus values in the previous Lemma.

Lemma 3.2 With the same notation as in (6), the following formulas hold:

$$
\begin{aligned}
& \sqrt{\sqrt{15}+\psi} \sqrt{\sqrt{15}-\psi}=\sqrt{2} \sqrt{\phi^{\star}}, \quad \sqrt{\sqrt{10}+\sqrt{\phi}} \sqrt{\sqrt{10}-\sqrt{\phi}}=\sqrt{\phi^{\star}}, \\
& \sqrt{\sqrt{15}+\psi^{\star}} \sqrt{\sqrt{15}-\psi^{\star}}=\sqrt{2} \sqrt{\phi}, \quad \sqrt{\sqrt{10}+\sqrt{\phi^{\star}}} \sqrt{\sqrt{10}-\sqrt{\phi^{\star}}}=\sqrt{\phi}, \\
& \sqrt{\sqrt{15} \pm \psi} \sqrt{\sqrt{15} \pm \psi^{\star}}=\frac{\sqrt{\phi^{\star}}}{\sqrt{2}}(\psi \pm \sqrt{3}), \quad \sqrt{\sqrt{10} \pm \sqrt{\phi}} \sqrt{\sqrt{10} \pm \sqrt{\phi^{\star}}}=\psi \pm \sqrt{5}, \\
& \sqrt{\sqrt{15} \pm \psi} \sqrt{\sqrt{15} \mp \psi^{\star}}=\frac{\sqrt{\phi}}{\sqrt{2}}\left(\sqrt{3} \pm \psi^{\star}\right), \quad \sqrt{\sqrt{10} \pm \sqrt{\phi}} \sqrt{\sqrt{10} \mp \sqrt{\phi^{\star}}}=\sqrt{5} \pm \psi^{\star}, \\
& \frac{\psi}{\sqrt{5}}\left(\sqrt{15} \pm \psi^{\star}\right)=\sqrt{3} \psi \pm 1, \quad \frac{\psi^{\star}}{\sqrt{5}}(\sqrt{15} \pm \psi)=\sqrt{3} \psi^{\star} \pm 1, \\
& \sqrt{\sqrt{15} \pm \psi} \sqrt{\sqrt{10} \pm \sqrt{\phi}}=\frac{\phi^{\star}}{2^{7 / 4} 5^{1 / 4}} \sqrt{\sqrt{3}-1} \sqrt{\sqrt{5}+\sqrt{3}}(\sqrt{3}+2 \pm \psi), \\
& \sqrt{\sqrt{15} \mp \psi} \sqrt{\sqrt{10} \pm \sqrt{\phi}}=\frac{\phi^{\star}}{2^{7 / 4} 5^{1 / 4}} \sqrt{\sqrt{3}+1} \sqrt{\sqrt{5}-\sqrt{3}}(\psi \mp \sqrt{3} \pm 2), \\
& \sqrt{\sqrt{15} \pm \psi^{\star}} \sqrt{\sqrt{10} \pm \sqrt{\phi^{\star}}}=\frac{\phi}{2^{7 / 4} 5^{1 / 4}} \sqrt{\sqrt{3}+1} \sqrt{\sqrt{5}+\sqrt{3}}\left(\psi^{\star} \mp \sqrt{3} \pm 2\right) \text {, } \\
& \sqrt{\sqrt{15} \mp \psi^{\star}} \sqrt{\sqrt{10} \pm \sqrt{\phi^{\star}}}=\frac{\phi}{2^{7 / 4} 5^{1 / 4}} \sqrt{\sqrt{3}-1} \sqrt{\sqrt{5}-\sqrt{3}}\left(\sqrt{3}+2 \pm \psi^{\star}\right) \text {, } \\
& 2 \sqrt{2} \pm \sqrt{3}+1=(\sqrt{2}+1)(\sqrt{3} \mp 1)(\sqrt{3} \pm \sqrt{2}) \\
& 2 \sqrt{2} \pm \sqrt{3}-1=(\sqrt{2}-1)(\sqrt{3} \pm 1)(\sqrt{3} \mp \sqrt{2}) \text {, } \\
& 2 \sqrt{3} \pm \sqrt{5} \pm 1=\frac{\phi^{\star}}{2 \sqrt{5}}(\sqrt{3} \pm 1)(\sqrt{5} \pm \sqrt{3}) \text {, } \\
& 2 \sqrt{3} \pm \sqrt{5} \mp 1=\frac{\phi}{2 \sqrt{5}}(\sqrt{3} \mp 1)(\sqrt{5} \pm \sqrt{3}) .
\end{aligned}
$$

Formulas with,$\pm \mp$ signs represent two identities, which can be read by taking the upper signs or the lower signs respectively.

Proof. The identities can be proved by direct manipulation.

The following Lemma is important for proving independence of the gamma values in (5) with respect to the standard equations.

Lemma 3.3 Let $N$ denote an integer greater than 2, and let $\varphi(N)$ denote Euler's totient value. Let $\Sigma$ be the set of gamma values at rational points with the denominator dividing $N$. Suppose that $\Sigma_{0}$ is a minimal subset of $\Sigma$ which determines all other gamma values in $\Sigma$ by the standard equations. Then $\Sigma_{0}$ has precisely $\varphi(N) / 2$ elements. 
Proof. This is a reformulation of Kubert's theorem in $\mathrm{Ku}$. See also [La, Chapter 2].

In the setting of the last Lemma, the set $\left\{\Gamma\left(\frac{k}{N}\right) \mid \operatorname{gcd}(k, N)=1, k<\frac{N}{2}\right\}$ looks like a natural candidate for $\Sigma_{0}$. This is a false impression in general. One can check the formulas in Section 2 and notice that for $N=20,24,30,60$ gamma values at rational points with the denominator $N$ depend on fewer gamma values of (5) than $\varphi(N) / 2$. In these cases, gamma values in the mentioned set are dependent.

Here we prove our main results. Along the way, we complement the set in (5) to a generating set for gamma values at rational points with the denominator dividing 120 . Note that 120 is the lowest common multiple of 24 and 60 .

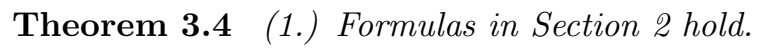

(2.) Standard equations (11)-(3) imply that any gamma value with the denominator dividing 120 can be expressed in terms of (5) and the following 6 gamma values:

$$
\Gamma\left(\frac{1}{40}\right), \quad \Gamma\left(\frac{3}{40}\right), \quad \Gamma\left(\frac{7}{40}\right), \quad \Gamma\left(\frac{1}{120}\right), \quad \Gamma\left(\frac{7}{120}\right), \quad \Gamma\left(\frac{11}{120}\right) .
$$

(3.) Standard equations (11)-(3) do not imply any relations ${ }^{1}$ between gamma values in (5).

Proof. For $x \in \mathbf{Q}$, let $R(x)$ denote reflection formula (2), and let $M_{n}(x)$ denote multiplication formula (3). Consider the following sequence of formulas:

$$
\begin{array}{r}
R\left(\frac{1}{2}\right), R\left(\frac{1}{3}\right), R\left(\frac{1}{4}\right), R\left(\frac{1}{5}\right), R\left(\frac{2}{5}\right), M_{2}\left(\frac{1}{6}\right), R\left(\frac{1}{6}\right), M_{2}\left(\frac{1}{8}\right), R\left(\frac{1}{8}\right), R\left(\frac{3}{8}\right), \\
M_{2}\left(\frac{1}{5}\right), M_{2}\left(\frac{1}{10}\right), R\left(\frac{1}{10}\right), R\left(\frac{3}{10}\right), M_{3}\left(\frac{1}{12}\right), M_{2}\left(\frac{1}{12}\right), R\left(\frac{1}{12}\right), R\left(\frac{5}{12}\right), \\
M_{3}\left(\frac{1}{15}\right), R\left(\frac{1}{15}\right), R\left(\frac{4}{15}\right), M_{5}\left(\frac{1}{15}\right), M_{3}\left(\frac{2}{15}\right), R\left(\frac{2}{15}\right), R\left(\frac{7}{15}\right), \\
M_{2}\left(\frac{1}{20}\right), R\left(\frac{1}{20}\right), R\left(\frac{9}{20}\right), M_{5}\left(\frac{1}{20}\right), M_{2}\left(\frac{3}{20}\right), R\left(\frac{3}{20}\right), R\left(\frac{7}{20}\right), \\
M_{3}\left(\frac{1}{24}\right), M_{2}\left(\frac{1}{24}\right), M_{2}\left(\frac{5}{24}\right), R\left(\frac{1}{24}\right), R\left(\frac{5}{24}\right), R\left(\frac{7}{24}\right), R\left(\frac{11}{24}\right), \\
M_{2}\left(\frac{1}{15}\right), M_{2}\left(\frac{2}{15}\right), M_{2}\left(\frac{1}{30}\right), M_{2}\left(\frac{7}{30}\right), R\left(\frac{1}{30}\right), R\left(\frac{7}{30}\right), R\left(\frac{11}{30}\right), R\left(\frac{13}{30}\right), \\
M_{3}\left(\frac{1}{60}\right), M_{3}\left(\frac{7}{60}\right), M_{2}\left(\frac{1}{60}\right), M_{2}\left(\frac{7}{60}\right), M_{2}\left(\frac{11}{60}\right), R\left(\frac{13}{60}\right), M_{2}\left(\frac{13}{60}\right), \\
R\left(\frac{1}{60}\right), R\left(\frac{7}{60}\right), R\left(\frac{11}{60}\right), R\left(\frac{17}{60}\right), R\left(\frac{19}{60}\right), R\left(\frac{23}{60}\right), R\left(\frac{29}{60}\right) .
\end{array}
$$

\footnotetext{
${ }^{1}$ This statement also holds for the total set of 16 gamma values in (5) and (10).
} 
We claim that these formulas, seen as equations in gamma values from the set (4), have a unique solution if the gamma values in (5) are assumed to be known. To prove this claim, note that the first 14 formulas, up till $R\left(\frac{3}{10}\right)$, determine consequently the gamma values at

$$
\frac{1}{2}, \frac{2}{3}, \frac{3}{4}, \frac{4}{5}, \frac{3}{5}, \frac{1}{6}, \frac{5}{6}, \frac{5}{8}, \frac{7}{8}, \frac{3}{8}, \frac{7}{10}, \frac{1}{10}, \frac{9}{10}, \frac{3}{10} .
$$

Each of those 14 formulas relates the respective gamma value to earlier concluded gamma values and the gamma values in (5), like in the proof of Lemma 3.1. The next 4 formulas, up till $R\left(\frac{5}{12}\right)$, express the products $\Gamma\left(\frac{1}{12}\right) \Gamma\left(\frac{5}{12}\right), \Gamma\left(\frac{1}{12}\right) \Gamma\left(\frac{7}{12}\right), \Gamma\left(\frac{1}{12}\right) \Gamma\left(\frac{11}{12}\right), \Gamma\left(\frac{5}{12}\right) \Gamma\left(\frac{7}{12}\right)$ in terms of gamma values in (5) and at the points in (11). Straightforward manipulation of those 4 formulas expresses the gamma values at $\frac{1}{12}, \frac{5}{12}, \frac{7}{12}, \frac{11}{12}$ in terms of (5D) and the earlier values. Similarly, the next 3 formulas, up till $R\left(\frac{4}{15}\right)$, consequently determine $\Gamma\left(\frac{11}{15}\right)$, $\Gamma\left(\frac{14}{15}\right), \Gamma\left(\frac{4}{15}\right)$; and then the subsequent 4 formulas all together determine $\Gamma\left(\frac{2}{15}\right), \Gamma\left(\frac{7}{15}\right)$, $\Gamma\left(\frac{8}{15}\right), \Gamma\left(\frac{13}{15}\right)$. In the same way, the next formulas $M_{2}\left(\frac{1}{20}\right), R\left(\frac{1}{20}\right), R\left(\frac{9}{20}\right)$ consequently determine $\Gamma\left(\frac{11}{20}\right), \Gamma\left(\frac{19}{20}\right), \Gamma\left(\frac{9}{20}\right)$; and then the following 4 formulas ${ }^{2}$ all together determine $\Gamma\left(\frac{3}{20}\right), \Gamma\left(\frac{7}{20}\right), \Gamma\left(\frac{13}{20}\right), \Gamma\left(\frac{17}{20}\right)$. The remaining formulas, starting from $M_{3}\left(\frac{1}{24}\right)$, consequently determine gamma values at

$$
\begin{aligned}
& \frac{17}{24}, \frac{13}{24}, \frac{5}{24}, \frac{23}{24}, \frac{19}{24}, \frac{7}{24}, \frac{11}{24}, \frac{17}{30}, \frac{19}{30}, \frac{1}{30}, \frac{7}{30}, \frac{29}{30}, \frac{23}{30}, \frac{11}{30}, \frac{13}{30}, \\
& \frac{41}{60}, \frac{47}{60}, \frac{31}{60}, \frac{37}{60}, \frac{11}{60}, \frac{13}{60}, \frac{43}{60}, \frac{59}{60}, \frac{53}{60}, \frac{49}{60}, \frac{17}{60}, \frac{19}{60}, \frac{23}{60}, \frac{29}{60} .
\end{aligned}
$$

We see that formulas are independent, and that they unique determine the mentioned gamma values. Also, the gamma values in (515) and the consequently concluded gamma values exhaust all elements of the set in (4). The claim follows.

To prove the first statement of the theorem, it is enough now to check that evaluations in Section 2 are compatible with the sequence of formulas introduced at the beginning of this proof. Identities in Lemmas 3.1 and 3.2 are very helpful in these computations.

Here we show the second statement of the Theorem. We need to check the gamma values at rational points with the denominators 40 and 120 . The equations

$$
M_{2}\left(\frac{1}{40}\right), M_{2}\left(\frac{3}{40}\right), M_{2}\left(\frac{7}{40}\right), R\left(\frac{19}{40}\right), M_{5}\left(\frac{3}{40}\right), M_{2}\left(\frac{11}{40}\right)
$$

determine consequently the gamma values at $\frac{21}{40}, \frac{23}{40}, \frac{27}{40}, \frac{19}{40}, \frac{11}{40}, \frac{31}{40}$ in terms of (15) and (10). After this, other gamma values at rational points with the denominator 40 are determined by reflection formulas. Similarly, the equations

$$
\begin{array}{r}
M_{3}\left(\frac{1}{120}\right), M_{3}\left(\frac{7}{120}\right), M_{3}\left(\frac{11}{120}\right), M_{2}\left(\frac{1}{120}\right), M_{2}\left(\frac{7}{120}\right), M_{2}\left(\frac{11}{120}\right), M_{2}\left(\frac{31}{120}\right), \\
M_{2}\left(\frac{41}{120}\right), M_{2}\left(\frac{47}{120}\right), R\left(\frac{59}{120}\right), M_{5}\left(\frac{11}{120}\right), M_{3}\left(\frac{1}{40}\right), M_{2}\left(\frac{23}{120}\right), M_{2}\left(\frac{43}{120}\right)
\end{array}
$$

\footnotetext{
${ }^{2}$ Gamma values cannot be determined entirely consequently. As was noted in $[\mathrm{Da}$, some relations between gamma values are implied by the standard equations up to taking square roots of both sides of an equality.
} 
determine consequently the gamma values at

$$
\frac{41}{120}, \frac{47}{120}, \frac{91}{120}, \frac{61}{120}, \frac{67}{120}, \frac{71}{120}, \frac{31}{120}, \frac{101}{120}, \frac{107}{120}, \frac{59}{120}, \frac{83}{120}, \frac{43}{120}, \frac{23}{120}, \frac{103}{120} .
$$

After this, other gamma values at rational points with the denominator 120 are determined by reflection formulas.

Now we show the third statement of the Theorem. We apply Lemma 3.3 with $N=120$ and conclude that the generating set of 16 gamma values in (5) and (10) cannot be made smaller. Therefore the standard equations do not imply any relations between those 16 values. This certainly applies to the subset (5) of gamma values.

Explicit expressions of Chapter 2 and of all gamma values at rational points with the denominator dividing 120, in terms of (5) and (10), are available as small Mathematica and Maple packages via webpage [KTV]. In those packages, the list (8) of algebraic numbers for multiplicative expressions of the gamma values is extended by

$$
\sqrt{6} \pm \sqrt{5}, \quad \sqrt{10} \pm 3, \quad \sqrt{\phi} \pm \sqrt{5}, \quad \sqrt{5} \pm \sqrt{\phi^{\star}}, \quad \sqrt{\phi} \pm \sqrt{3}, \quad \sqrt{3} \pm \sqrt{\phi^{\star}} .
$$

Here we discuss briefly algebraic independence of gamma values. Rohrlich's conjecture Wa2, Section 3.3] implies that all multiplicative relations between gamma values at rational points are implied by the standard equations. The stronger Lang's conjecture [La, Ch. 2] implies that all algebraic relations between gamma values at rational points are implied by the standard equations. If these conjectures are true, we have (respectively) multiplicative or algebraic independence of the distinguished gamma values in (5). Analogues of these conjectures are proved for the Thakur's gamma function in positive characteristic [ABP. It is known that all $\mathbf{Q}$-linear relations between the beta values $\mathrm{B}(a, b)$ with $a, b, a+b \in \mathbf{Q} \backslash \mathbf{Z}$ are implied by the standard equations [WW. Chudnovsky proved that $\Gamma\left(\frac{1}{3}\right)$ and $\Gamma\left(\frac{1}{4}\right)$ are transcendental over $\mathbf{Q}(\pi)$; see Wa1. Algebraicity of gamma terms is determined, assuming Rohrlich's conjecture, by Koblitz-Ogus criterion [KO].

\section{Application to algebraic hypergeometric functions}

It is well-known that Gauss hypergeometric functions can be evaluated at some points in terms of the gamma function. The most important formula is the Gauss' identity AAR Theorem 2.2.2]:

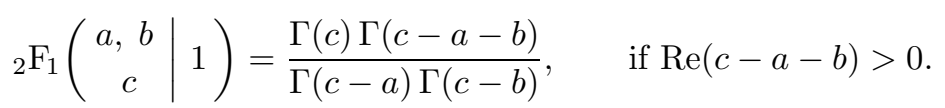

Akin to this identity, linear relations between the 24 Kummer's solutions of the hypergeometric differential equation (and analytic continuation formulas for the Gauss hypergeometric 
function) involve similar gamma terms. An example is AAR, Corollary 2.3.3]:

$$
\begin{aligned}
{ }_{2} \mathrm{~F}_{1}\left(\begin{array}{c|c}
a, b \\
c
\end{array} \mid x\right)= & \frac{\Gamma(c) \Gamma(c-a-b)}{\Gamma(c-a) \Gamma(c-b)}{ }_{2} \mathrm{~F}_{1}\left(\begin{array}{c}
a, b \\
a+b+1-c
\end{array} \mid 1-x\right) \\
& +\frac{\Gamma(c) \Gamma(a+b-c)}{\Gamma(a) \Gamma(b)}(1-x)^{c-a-b}{ }_{2} \mathrm{~F}_{1}\left(\begin{array}{c}
c-a, c-b \\
c+1-a-b
\end{array} \mid 1-x\right) .
\end{aligned}
$$

Our formulas in Section 2 can be used to evaluate widely used instances of the Gauss hypergeometric function most explicitly. In particular, evaluation of algebraic Gauss hypergeometric functions with (13) requires gamma values at rational points with the denominator dividing 24 or 60 ; see $\underline{\mathrm{Sch}}, \underline{\mathrm{Ka}}$, $\underline{\mathrm{Vi}}$. The formulas in Section 2 are precisely sufficient to evaluate algebraic Gauss hypergeometric functions at $x=1$. Here are a few examples:

$$
\begin{aligned}
& { }_{2} \mathrm{~F}_{1}\left(\begin{array}{c|c}
\frac{1}{4},-\frac{1}{12} & 1 \\
\frac{2}{3} & 1
\end{array}\right)=\frac{\Gamma\left(\frac{1}{2}\right) \Gamma\left(\frac{2}{3}\right)}{\Gamma\left(\frac{3}{4}\right) \Gamma\left(\frac{5}{12}\right)}=\frac{\sqrt{\sqrt{3}+1}}{2^{1 / 4} 3^{3 / 8}}, \\
& { }_{2} \mathrm{~F}_{1}\left(\begin{array}{c|c}
\frac{5}{24},-\frac{1}{24} & 1 \\
\frac{2}{3} &
\end{array}\right)=\frac{\Gamma\left(\frac{1}{2}\right) \Gamma\left(\frac{2}{3}\right)}{\Gamma\left(\frac{11}{24}\right) \Gamma\left(\frac{17}{24}\right)}=\frac{\sqrt{\sqrt{3}+\sqrt{2}}}{2^{1 / 12} \sqrt{3}}, \\
& { }_{2} \mathrm{~F}_{1}\left(\begin{array}{c|c}
\frac{11}{60},-\frac{1}{60} & 1 \\
\frac{2}{3} & 1
\end{array}\right)=\frac{\Gamma\left(\frac{1}{2}\right) \Gamma\left(\frac{2}{3}\right)}{\Gamma\left(\frac{29}{60}\right) \Gamma\left(\frac{41}{60}\right)}=\frac{\sqrt{\phi^{\star}} \sqrt{\sqrt{3}+1} \sqrt{\sqrt{5}+\sqrt{3}}}{2 \sqrt{3} 5^{7 / 24}}, \\
& { }_{2} \mathrm{~F}_{1}\left(\begin{array}{c|c}
\frac{3}{10},-\frac{1}{30} & 1 \\
\frac{3}{5} & 1
\end{array}\right)=\frac{\Gamma\left(\frac{1}{3}\right) \Gamma\left(\frac{3}{5}\right)}{\Gamma\left(\frac{3}{10}\right) \Gamma\left(\frac{19}{30}\right)}=\frac{\sqrt{\sqrt{15}+\psi}}{2^{19 / 30} 3^{1 / 20} 5^{1 / 3}} .
\end{aligned}
$$

More of these evaluations are independently presented in [Ka]. Explicit expressions for algebraic Gauss hypergeometric functions are given in $\mathrm{Vi}$.

\section{Gamma values and (hyper)elliptic integrals}

As is known [BZ, some gamma values can be expressed in terms of special values of the elliptic K-function (i.e., complete elliptic integral of the first kind). The advantage of doing this is that numerical values of the elliptic $\mathbf{K}$-function can be very effectively computed using the arithmetic-geometric mean; see [AAR, Section 3.2] and [BB pg. 137].

Here we present direct expressions for most of gamma values from (5) in terms of the elliptic K-function. By combining the data in WR, pages EllipticIntegralSingularValue.html, EllipticLambdaFunction.html] and our formulas in Section [2 we get the following evaluations:

$$
\begin{aligned}
& \Gamma\left(\frac{1}{3}\right)=\frac{\pi^{1 / 3} 2^{7 / 9}}{3^{1 / 12}} \mathbf{K}\left(\frac{\sqrt{3}-1}{2 \sqrt{2}}\right)^{1 / 3}, \\
& \Gamma\left(\frac{1}{4}\right)=2 \pi^{1 / 4} \mathbf{K}\left(\frac{1}{\sqrt{2}}\right)^{1 / 2}, \\
& \Gamma\left(\frac{1}{8}\right)=\pi^{1 / 8} 2^{17 / 8} \mathbf{K}\left(\frac{1}{\sqrt{2}}\right)^{1 / 4} \mathbf{K}(\sqrt{2}-1)^{1 / 2},
\end{aligned}
$$




$$
\begin{aligned}
\Gamma\left(\frac{1}{15}\right)= & \frac{\pi^{1 / 6} 3^{29 / 60} 5^{1 / 24} \sqrt{\phi^{\star}} \sqrt{\psi+\sqrt{3}}}{2^{1 / 9}} \Gamma\left(\frac{1}{5}\right)^{1 / 2} \Gamma\left(\frac{2}{5}\right)^{-1 / 2} \mathbf{K}\left(\frac{\sqrt{3}-1}{2 \sqrt{2}}\right)^{1 / 6} \\
& \times \mathbf{K}\left(\frac{(2-\sqrt{3})(3-\sqrt{5})(\sqrt{5}-\sqrt{3})}{8 \sqrt{2}}\right)^{1 / 2}, \\
\Gamma\left(\frac{1}{20}\right)= & \frac{2^{9 / 40} 5^{1 / 8} \phi^{5 / 8} \sqrt{\psi^{\star}+1}}{\pi^{1 / 4}} \Gamma\left(\frac{1}{5}\right)^{1 / 2} \Gamma\left(\frac{2}{5}\right)^{1 / 2} \mathbf{K}\left(\sqrt{\frac{1}{2}-\sqrt{\sqrt{5}-2}}\right)^{1 / 2}, \\
\Gamma\left(\frac{1}{24}\right)= & \pi^{1 / 24} 2^{89 / 36} 3^{25 / 48} \sqrt{\sqrt{2}+1}(\sqrt{3}-1)^{1 / 4} \mathbf{K}\left(\frac{1}{\sqrt{2}}\right)^{1 / 4} \mathbf{K}\left(\frac{\sqrt{3}-1}{2 \sqrt{2}}\right)^{1 / 3} \\
& \times \mathbf{K}((2-\sqrt{3})(\sqrt{3}-\sqrt{2}))^{1 / 2} .
\end{aligned}
$$

Other gamma values in (5) can be expressed in terms of hyperelliptic integrals. For example, consider the integrals:

$$
H_{1}=\int_{0}^{1} \frac{d z}{\sqrt{1-z^{5}}}, \quad H_{2}=\int_{0}^{1} \frac{z d z}{\sqrt{1-z^{5}}} .
$$

Note that the two differentials under integration form a basis for the space of holomorphic differentials on the genus 2 curve $y^{2}=x^{5}-1$. We have $\mathrm{B}\left(\frac{1}{5}, \frac{1}{2}\right)=5 H_{1}, \mathrm{~B}\left(\frac{2}{5}, \frac{1}{2}\right)=5 H_{2}$; this can be seen after the substitution $t \mapsto z^{5}$ in the standard definition [AAR, Definition 1.1.3] of the beta integral. From the two beta values we conclude

$$
\Gamma\left(\frac{1}{5}\right)=\pi^{1 / 5} 2^{19 / 50} \sqrt{5} \phi^{1 / 10} H_{1}^{2 / 5} H_{2}^{1 / 5}, \quad \Gamma\left(\frac{2}{5}\right)=\pi^{2 / 5} 2^{4 / 25} \phi^{1 / 5} H_{1}^{-1 / 5} H_{2}^{2 / 5} .
$$

To get similar expressions for gamma values at rational points with the denominator 60 , one may substitute $t \mapsto z^{30}$ into the beta integrals $\mathrm{B}\left(\frac{1}{60}, \frac{1}{2}\right)$ and $\mathrm{B}\left(\frac{7}{60}, \frac{1}{2}\right)$ and get hyperelliptic integrals on the genus 15 curve $y^{2}=x^{31}-x$. Possibly, there are integrals on lower genus curves related to those gamma values.

\section{References}

[AAR] G.E. Andrews, R. Askey, R. Roy, Special Functions, Cambridge Univ. Press, Cambridge, 1999.

[ABP] G.W. Anderson, W.D. Brownawell, M.A. Papanikolas, Determination of the algebraic relations among special $\Gamma$-values in positive charateristic, accepted by Annals of Mathematics; available at http://arxiv.org/abs/math.NT/0207168

[BB] J.M. Borwein, D.H. Bailey, Mathematics by Experiment; Plausible Reasoning in the 21st Century, A.K. Peters, Natick Ma, 2003.

[BZ] J.M. Borwein, I.J. Zucker, Fast evaluation of the gamma function for small rational fractions using complete elliptic integrals of the first kind, IMA J. of Numer Analysis, 12(1992), pg. $519-526$. 
[Ca] R. Campbell, Les intégrales eulériennes et leurs applications, Dunod, Paris, 1966.

[Da] P. Das, Algebraic gamma monomials and double coverings of cyclotomic fields, Trans. Amer. Math. Soc., Vol. 352, No. 8 (2000), pg. 3557-3594.

[De] P. Deligne, Valuers de fonctions L et périodes d'intégrales, Proc. Sympos. Pure Math., 33 (1979), part 2, pg. 313-346.

[Ka] M. Kato, Connection formulas for algebraic hypergeometric functions, preprint of Ryukyus Univ., 2004; submitted to the Kyushu Journal of Mathematics.

[KO] N. Koblitz, A. Ogus, Algebraicity of some products of values of the $\Gamma$ function, Appendix to the reference De, pg. 343-346.

[Koo] J. Kool, Gamma Values, website http://www.jgk.org/math/gamma-values.html

[KTV] T. H. Koornwinder, N. Temme, R. Vidunas, Algorithmic Methods for Special Functions by Computer Algebra, NWO research project 613-06-565 website, http://staff.science.uva.nl/ ${ }^{\sim}$ thk/specfun/compalg.html

$[\mathrm{Ku}]$ D. Kubert, The universal ordinary distribution, Bull. Soc. Math. France, 107 (1979), pg. 179-202.

[KZ] M. Kontsevich, D. Zagier, Periods, in B. Engquist, W. Schmid (Ed.) Mathematics Unlimited2001 and Beyond, Springer, Berlin, 2001, pg. 771-808.

[La] S. Lang, Cyclotomic Fields I and II, Graduate Texts in Mathematics, No 121, SpringerVerlag, New York 1990.

[SC] A. Selberg, S. Chowla, On Epstein's zeta function, J. Reine Angew. Math., 227 (1967), pg. 86-110.

[Sch] H. Schwarz, Ueber diejenigen Falle, in welchen die Gaussische hypergeometrische Reihe eine algebraische Funktion ihres vierten Elements darstelt, J. Reine Angew. Math., 75 (1872), pg. 292-335.

[Vi] R. Vidunas, Darboux evaluations of algebraic Gauss hypergeometric functions, preprint, Kyushu university, 2004.

[Wa1] M. Waldschmidt, Les travaux de G.V. Chudnovsky sur les nombres transcendants, in Séminaire Bourbaki, Vol. 1975/76, 28e année, Exp. No 488, pg. 274-292; Lecture Notes in Math., Vol. 567, Springer, Berlin, 1977.

[Wa2] M. Waldschmidt, Open Diophantine Problems, to appear in Moscow Mathematical Journal Vol. 4 No. 1 (2004); available at http://arxiv.org/math.NT/0312440

[WR] Wolfram Research Mathworld website, http://mathworld.wolfram.com

[WW] J. Wolfart, G. Wüstholz, Der Überlagerungradius gewisser algerbaischer Kurven und die Werte de Betafunktion an rationalen Stellen, Math. Ann. 273 (1985), pg. 1-15.

[Zu] I.J. Zucker, The evaluation in terms of $\Gamma$-functions of the periods of elliptic curves admitting complex multiplication, Proc. Camb. Phil. Soc, 82 (1977), pg. 111-118. 